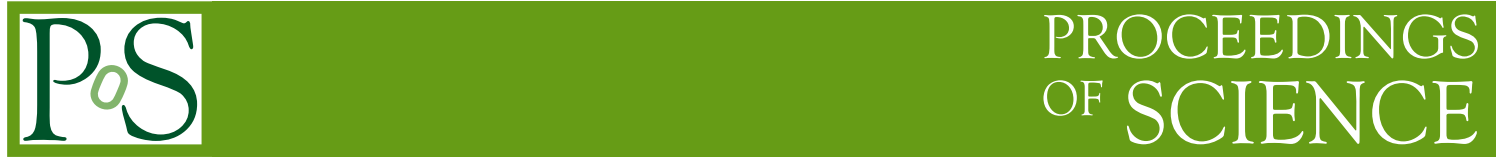

\title{
Observation of $\eta_{b}(2 S)$ in $\Upsilon(2 S) \rightarrow \gamma \eta_{b}(2 S)$, $\eta_{b}(2 S) \rightarrow$ hadrons, and Confirmation of $\eta_{b}(1 S)$
}

\section{Kamal K. Seth*}

Northwestern University, Evanston, IL 60208 (USA)

E-mail: kseth@northwestern.edu

The data for 9.3 million $\Upsilon(2 S)$ and 20.9 million $\Upsilon(1 S)$ taken with the CLEO III detector has been used to study the radiative population of states identified by their decay into twenty six different exclusive hadronic final states. In the $\Upsilon(2 S)$ decays an enhancement is observed at $\mathrm{a} \sim 5 \sigma$ level at a mass of $9974.6 \pm 2.3$ (stat) \pm 2.1 (syst) $\mathrm{MeV}$. It is attributed to $\eta_{b}(2 S)$, and corresponds to the $\Upsilon(2 S)$ hyperfine splitting of $48.7 \pm 2.3$ (stat) \pm 2.1 (syst) $\mathrm{MeV}$. In the $\Upsilon(1 S)$ decays, the identification of $\eta_{b}(1 S)$ is confirmed at a $\sim 3 \sigma$ level with $M\left(\eta_{b}(1 S)\right)$ in agreement with its known value.

Xth Quark Confinement and the Hadron Spectrum,

October 8-12, 2012

TUM Campus Garching, Munich, Germany

\footnotetext{
${ }^{*}$ Speaker.
} 


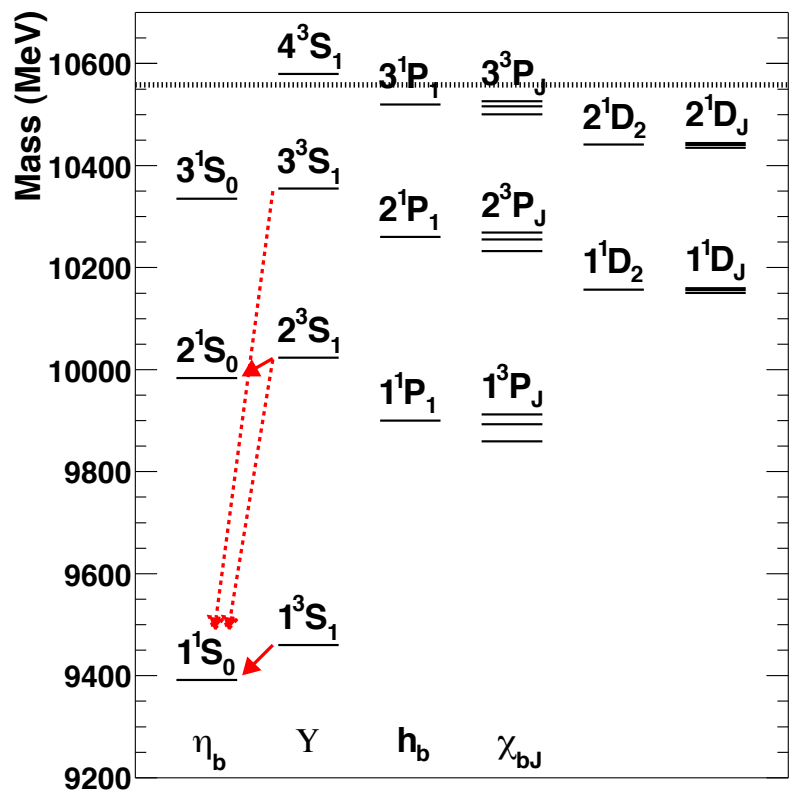

Figure 1: Level spectrum of bound states of the $b \bar{b}$ bottomonium system.

The spin-dependent interactions between quarks give rise to a rich spectrum of states of quarkonia, and the $\mid b \bar{b}>$ bottomonium is the preferred system to study these because the strong coupling constant, $\alpha_{s}\left(m_{b}\right)$ is small $(\sim 0.2)$, and relativistic effects are small $\left(\left(v^{2} / c^{2}\right) \sim 0.1\right)$. Among the spin-dependent interactions, spin-orbit, tensor, and spin-spin, the spin-spin or hyperfine interaction holds a special place. It splits a state into two, the spin-singlet, $S=\overrightarrow{s_{1}}+\overrightarrow{s_{2}}=0$, and the spin-triplet, $S=\overrightarrow{s_{1}}+\overrightarrow{s_{2}}=1$, and determines the ground-state masses of all hadrons. Indeed, the study of the hyperfine interaction in quarkonia by means of measuring the hyperfine mass splitting, and the radiatvie transition rates between the triplet and singlet states has been the subject of great experimental interest and development recently. It has also catalyzed a large number of theoretical efforts, both in terms of QCD-based models and lattice calculations.

There is a large asymmetry between the fortunes of spin-singlet and spin-triplet states of quarkonia. The S-wave spin-triplet states $\psi(c \bar{c})$ and $\Upsilon(b \bar{b})$ with $J^{P C}=1^{--}$are directly excited in $e^{+} e^{-}$annihilation via a virtual photon $\left(J^{P C}=1^{--}\right)$, and the P-wave triplet states $\chi(c \bar{c})$ and $\chi(b \bar{b})$ with $J^{P C}=(0,1,2)^{++}$are conviniently excited by strong E1 radiative transitions from them. The spin-singlet states $J^{P C}=0^{-+}(\eta), 1^{+-}(h)$ are not so blessed. They are neither directly excited, nor are fed by strong E1 radiative transitions from the triplet states. The triplet $\rightarrow$ singlet radiative transition is weak M1, called 'allowed' M1 when it takes place between states of the same principal quantum number, i.e., $\left|n^{3} L_{J}>\rightarrow\right| n^{1} L_{J}>\gamma$, and 'forbidden' when it takes place between states of different n, i.e., $\left|n^{\prime 3} L_{J}>\rightarrow\right| n^{1} L_{J}>\gamma\left(n^{\prime} \neq n\right)$. Fig. 1 illustrates both the 'allowed' M1 transitions (solid arrows) and the 'forbidden' M1 transitions (dashed arrows) for bottomonium.

As is well-known, in the lowest order the $q \bar{q}$ interaction is modeled as a central potential with a Coulombic $1 / r$ part, and a linear in $r$ confinement part. The Coulombic part is, of course, Lorentz 
vector, and gives rise to the familiar spin-orbit, tensor, and spin-spin potentials, and the confinement part, generally assumed to be Lorentz scalar, does not make any contribution to the long-range spin dependent potential. As a result, the 'allowed' M1 radiative transitions, $\left|n^{3} L_{J}>\rightarrow \gamma\right| n^{1} L_{J}>$, are theoretically simple to understand, being similar to those in positronium. For S-wave $q \bar{q}$ states, in the perturbative approximation,

$$
\begin{aligned}
\Delta M_{\mathrm{hf}}(n S)_{q \bar{q}}=M\left(n^{3} S_{1}\right)-M\left(n^{1} S_{0}\right) & =(32 \pi / 9) \alpha_{s}(q)|\psi(0)|_{q \bar{q}} / m_{q}^{2} \\
& =\left(8 \alpha_{s} / \alpha_{\mathrm{em}}^{2}\right) \Gamma\left({ }^{3} S_{1} \rightarrow e^{+} e^{-}\right)_{\exp } / \mathrm{CF},
\end{aligned}
$$

where the first order $\mathrm{QCD}$ radiative correction, $\mathrm{CF} \approx 0.66$. This leads to the predictions, $\Delta M_{\mathrm{hf}}(1 S)_{b \bar{b}}=$ $61 \mathrm{MeV}$, and $\Delta M_{\mathrm{hf}}(2 S)_{b \bar{b}}=28 \mathrm{MeV}$. It is interesting to note that relativistic corrections do not change these predictions. Godfrey and Isgur [1] obtain identical results in their 'relativised' pQCD model calculations, and so do Brambilla, Jia and Vairo [2] in their more sophisticated pNRQCD effective field theory calculations.

Similarly, the radiative widths for the $n \rightarrow n$ transitions are simply,

$$
\Gamma\left(\left|n^{3} S_{1}>\rightarrow \gamma\right| n^{1} S_{0}>\right)_{b \bar{b}}=\left(16 \alpha_{\mathrm{em}} / 27\right) E_{\gamma}^{3} / M^{2}(\Upsilon(1 S)),
$$

which leads to the predictions that the branching fractions, $\mathrm{BF}\left(\Upsilon(1 S) \rightarrow \gamma \eta_{b}(1 S)\right)=2 \times 10^{-4}$, and $\mathrm{BF}\left(\Upsilon(2 S) \rightarrow \gamma \eta_{b}(2 S)\right)=0.3 \times 10^{-4}$ for the above predicted values of $\Delta M_{\mathrm{hf}}\left(=E_{\gamma}\right)$.

In contrast, theoretical predictions for the 'forbidden' M1 transitions are notoriously difficult and unreliable. For example, both with and without relativistic corrections the potential model predictions for $\mathrm{BF}\left(\Upsilon(2 S) \rightarrow \gamma \eta_{b}(1 S)\right)$ vary between $0.05 \times 10^{-4}$ to $13 \times 10^{-4}$ as summarized by Godfrey and Rosner [3], and a pNRQCD prediction is $(250 \pm 160) \times 10^{-4}$ [2], whereas the experimental result is $\mathrm{BF}\left(\Upsilon(2 S) \rightarrow \gamma \eta_{b}(2 S)\right)=(3.9 \pm 1.6) \times 10^{-4}[4]$.

The experimental situation between 'allowed' and 'forbidden' M1 transitions is opposite of the theoretical one described above. As shown in Fig. 1, the transition photon energies for the 'allowed' transitions $\left|n^{3} S_{1}>\rightarrow\right| n^{1} S_{0}>\gamma$ are small, with $E_{\gamma}<100 \mathrm{MeV}$ for both n=1, and n=2, making it very difficult to make direct observation of these transition photons in presence of orders of magnitude larger low energy photon background. On the other hand, the difficult to understand 'forbidden' transitions have the advantage of large photon energy and the consequent $E_{\gamma}^{3}$ enhancement in width. As a result, the first successful observation of $\eta_{b}\left(1^{1} S_{0}\right)$ was made by observing the $\sim 908 \mathrm{MeV}$ photon for the $\Upsilon\left(3^{3} S_{1}\right) \rightarrow \eta_{b}\left(1^{1} S_{0}\right) \gamma$ 'forbidden' transition which has a factor $\sim 2000$ larger yield than a $70 \mathrm{MeV}$ 'allowed' transition. Even then, the task was not easy because of the nearby presence of even a factor 1000 larger transition to $\chi_{b J}\left(1^{3} P_{J}\right)$ states. The spectra for the $\mathrm{BaBar}$ [4] observation, and the CLEO [5] confirmation of $\eta_{b}(1 S)$ are shown in Fig. 2. The results for $\Delta M_{\mathrm{hf}}(1 S)_{b \bar{b}}=$ $71.4_{-4.1}^{+3.5} \mathrm{MeV}$ (BaBar), $\Delta M_{\mathrm{hf}}(2 S)_{b \bar{b}}=68.5 \pm 6.9 \mathrm{MeV}$ (CLEO) are in good agreement.

Because the $n \rightarrow n$ 'allowed' M1 transitions are theoretically more tractable we decided that we would attempt to identify them despite the difficulties mentioned. It was quite clear that since there was no hope of directly observing the $\sim 70 \mathrm{MeV}$ photons for the $\Upsilon\left(1^{3} S_{1}\right) \rightarrow \eta_{b}\left(1^{1} S_{0}\right) \gamma$ decay, or the $\leq 50 \mathrm{MeV}$ photons for the $\Upsilon\left(2^{3} S_{1}\right) \rightarrow \eta_{b}\left(2^{1} S_{0}\right) \gamma$ decay, we will have to tag them via the hadronic decays of $\eta_{b}(1 S)$ and $\eta_{b}(2 S)$. Individual hadronic decays of $\chi_{b 0}(1 P)$ are known to have branching fractions of the order $<10^{-4}$. We expect the branching fractions for hadronic decays of $\eta_{b}(1 S, 2 S)$ to be as weak, or weaker. So, we would require to identify as many hadronic decays as 

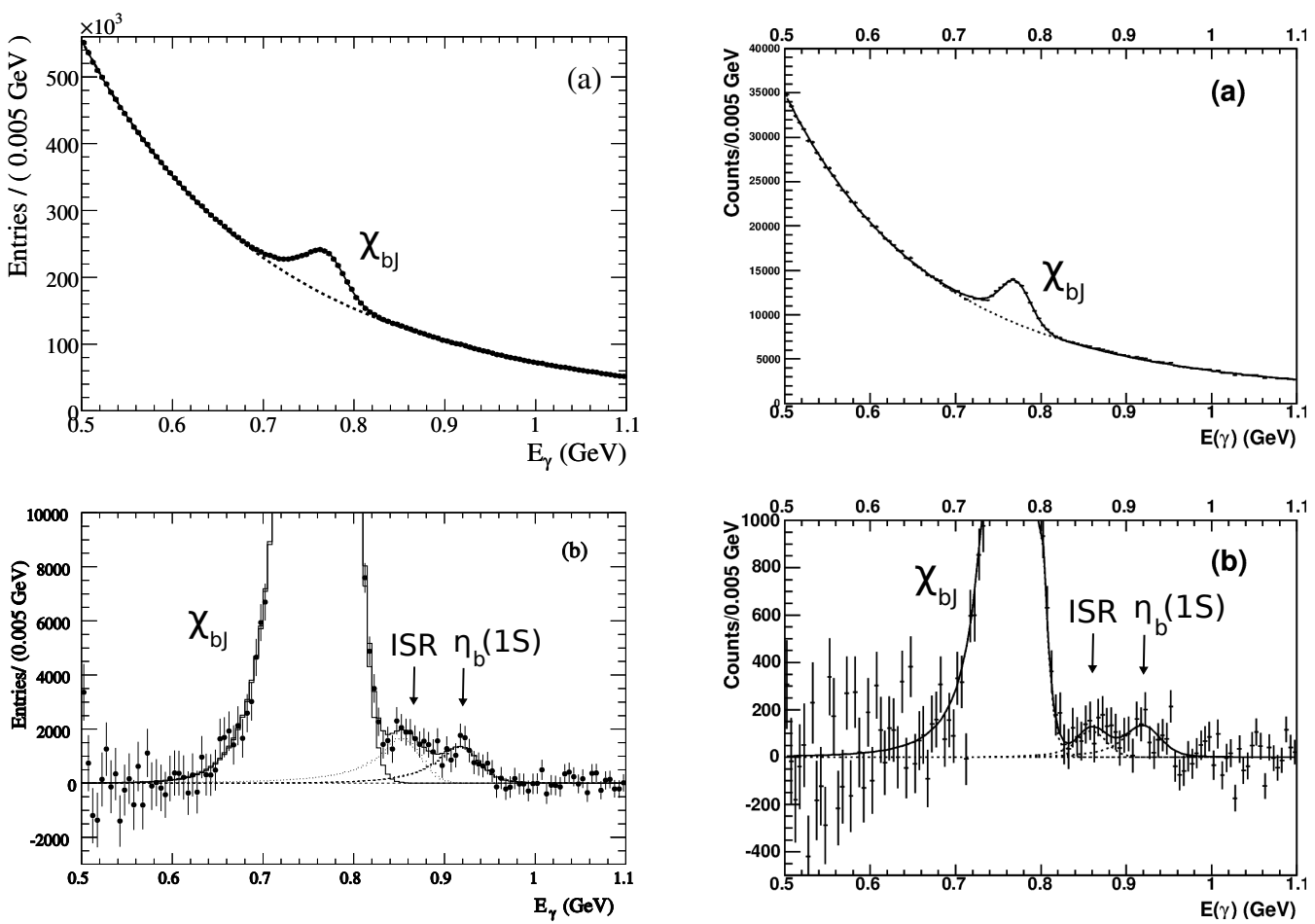

$$
\begin{gathered}
\text { BaBar }(2008) \\
\Upsilon(3 S) \rightarrow \gamma \eta_{b}(1 S) \\
\Delta M_{\mathrm{hf}}(1 S)_{b \bar{b}}=71.4_{-4.1}^{+3.5} \mathrm{MeV}
\end{gathered}
$$

$$
\begin{gathered}
\text { CLEO }(2010) \\
\Upsilon(3 S) \rightarrow \gamma \eta_{b}(1 S) \\
\Delta M_{\mathrm{hf}}(2 S)_{b \bar{b}}=68.5 \pm 6.9 \mathrm{MeV}
\end{gathered}
$$

Figure 2: Illustrating observation of $\eta_{b}(1 S)$ in the $E_{\gamma}=908 \mathrm{MeV}$ 'forbidden' M1 radiative decay, $\Upsilon(3 S) \rightarrow$ $\gamma \eta_{b}(1 S)$ by BaBar [4] and its confirmation by CLEO [5].

we could. We have done precisely that by reconstructing 26 different hadronic decay modes each of $\eta_{b}(1 S)$ and $\eta_{b}(2 S)$. These are listed in Table 1.

We analyze the data for 9.3 million $\Upsilon(2 S)$ and 20.9 million $\Upsilon(1 S)$ taken with the CLEO III detector. We select events with 4,6,8, or 10 charged particle tracks with zero net charge and at least one photon candidate. We identify the charged particles using $\mathrm{dE} / \mathrm{dx}$ and $\mathrm{RICH}$ information, and reconstruct the full event including both the charged hadrons and the transition photon by making a 4C kinematic fit with net momentum of zero. We further use a thrust angle cut of $\left|\cos \theta_{T}\right|<0.5$. The resulting mass distributions are shown in Fig. 3 as functions of $\Delta M \equiv M(\Upsilon(n S))-M$ (hadrons), which should show $\eta_{b}(n S)$ peaks directly. In these distributions at the smallest value of $\Delta M$ we observe the large, steeply falling contributions from the direct decays of $\Upsilon(n S)$. These are followed closely with small peaks which we attribute to $\eta_{b}(1 S)$ and $\eta_{b}(2 S)$. For $\Upsilon(2 S)$ peaks for the radiative transitions to $\chi_{b J}(1 P)$ states are also observed. The background levels are very small, particularly in the $\Upsilon(2 S)$ distribution, and there is no evidence for FSR contributions.

We have fitted the $\Delta M$ spectra of Fig. 3. The $\Upsilon(n S)$ direct decay contributions at small $\Delta M$ were fitted with simple exponentials, and the observed peaks were parametrized as Gaussians convolved with known widths of $\chi_{b J}(1 P)$ states, and the assumed values, $\Gamma\left(\eta_{b}(1 S)\right)=10 \mathrm{MeV}$ and 


\begin{tabular}{llll}
\hline $\boldsymbol{X}_{\boldsymbol{i}}=\mathbf{4}$ Track & $\boldsymbol{X}_{\boldsymbol{i}}=\mathbf{6}$ Track & $\boldsymbol{X}_{\boldsymbol{i}}=\mathbf{8}$ Track & $\boldsymbol{X}_{\boldsymbol{i}}=\mathbf{1 0}$ Track \\
\hline $2\left(\pi^{+} \pi^{-}\right)$ & $3\left(\pi^{+} \pi^{-}\right)$ & $4\left(\pi^{+} \pi^{-}\right)$ & $5\left(\pi^{+} \pi^{-}\right)$ \\
$2\left(K^{+} K^{-}\right)$ & $2\left(K^{+} K^{-}\right) \pi^{+} \pi^{-}$ & $2\left(K^{+} K^{-}\right) 2\left(\pi^{+} \pi^{-}\right)$ & $2\left(K^{+} K^{-}\right) 3\left(\pi^{+} \pi^{-}\right)$ \\
$K^{+} K^{-} \pi^{+} \pi^{-}$ & $K^{+} K^{-} 2\left(\pi^{+} \pi^{-}\right)$ & $K^{+} K^{-} 3\left(\pi^{+} \pi^{-}\right)$ & $K^{+} K^{-} 4\left(\pi^{+} \pi^{-}\right)$ \\
$p \bar{p} \pi^{+} \pi^{-}$ & $p \bar{p} 2\left(\pi^{+} \pi^{-}\right)$ & $p \bar{p} 3\left(\pi^{+} \pi^{-}\right)$ & $p \bar{p} 4\left(\pi^{+} \pi^{-}\right)$ \\
$K_{S} K^{ \pm} \pi^{\mp}$ & $p \bar{p} K^{+} K^{-} 2\left(\pi^{+} \pi^{-}\right)$ & $p \bar{p} K^{+} K^{-} 2\left(\pi^{+} \pi^{-}\right)$ & $p \bar{p} K^{+} K^{-} 3\left(\pi^{+} \pi^{-}\right)$ \\
& $K_{S} K^{ \pm} \pi^{\mp} \pi^{+} \pi^{-}$ & $K_{S} K^{ \pm} \pi^{\mp} 2\left(\pi^{+} \pi^{-}\right)$ & $K_{S} K^{ \pm} \pi^{\mp} 3\left(\pi^{+} \pi^{-}\right)$ \\
& $K_{S} K_{S} \pi^{+} \pi^{-}$ & $K_{S} K_{S} 2\left(\pi^{+} \pi^{-}\right)$ & $K_{S} K_{S} 3\left(\pi^{+} \pi^{-}\right)$ \\
\hline
\end{tabular}

Table 1: List of the 26 charged hadronic decays of $\eta_{b}(1 S)$ and $\eta_{b}(2 S)$ used in the present investigation.
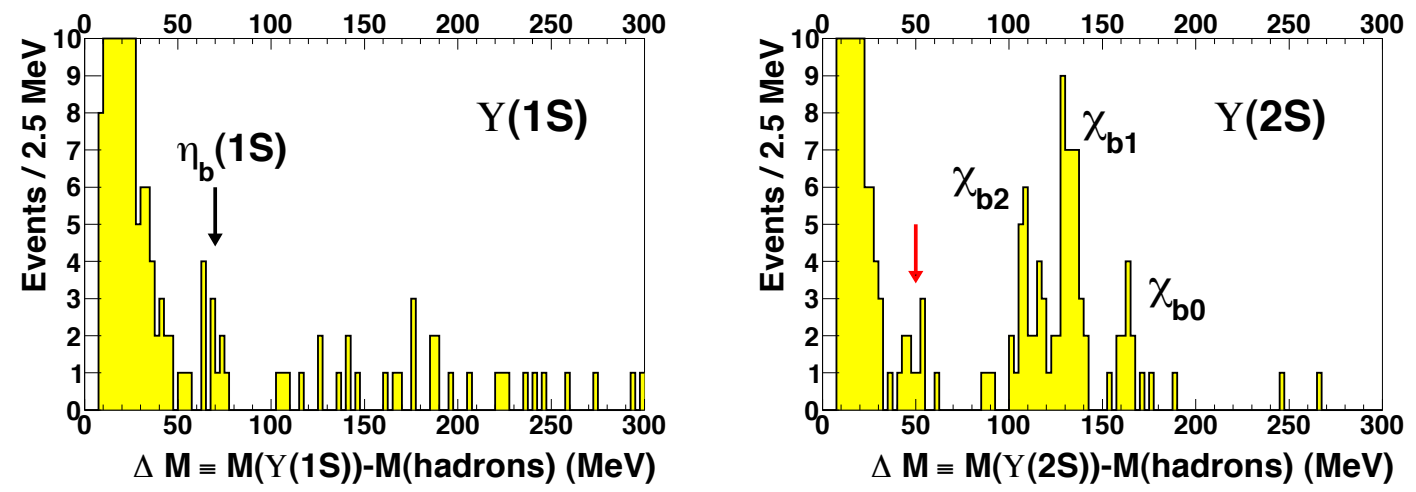

Figure 3: Observed mass distributions, $\Delta M=M(\Upsilon(n S))-M$ (hadrons) for $\Upsilon(1 S)$ (left) and $\Upsilon(2 S)$ (right), unbiassed by fits.

$\Gamma\left(\eta_{b}(2 S)\right)=5 \mathrm{MeV}$. The fits are shown in Fig. 4. Since the shapes of the $\Upsilon(n S)$ contributions at small $\Delta M$ are important in determining the significance of the $\eta_{b}(1 S, 2 S)$ peaks, we have tried various shapes for them, and found that simple exponentials fit best in both cases. To emphasize this point, in Fig. 4 we show the fits in semi-log plots as well. In both representations we find that the peaks at $\Delta M \approx 70 \mathrm{MeV}$ in the $\Upsilon(1 S)$ distribution and at $\Delta M \approx 50 \mathrm{MeV}$ in the $\Upsilon(2 S)$ distribution are well resolved from the $\Upsilon(n S)$ direct contributions.

Our results are shown in Table 2. For $\eta_{b}(1 S)$ our result, $M\left(\eta_{b}(1 S)\right)=9393.2 \pm 3.4 \pm 2.3 \mathrm{MeV}$ agrees well with the results of BaBar [4] and CLEO [5], and the PDG2012 average of $9391.0 \pm$ $2.8 \mathrm{MeV}$. This agreement is very reassuring for our method of analysis. Our result corresponds to $\Delta M_{\mathrm{hf}}(1 S)_{b \bar{b}}=67.1 \pm 3.4 \pm 2.3 \mathrm{MeV}$. For $\eta_{b}(2 S)$ our result represents the first observation of $\eta_{b}(2 S)$ at a significance level of 4.9. We find $M\left(\eta_{b}(2 S)\right)=9974.6 \pm 2.3 \pm 2.1 \mathrm{MeV}$. It corresponds to $\Delta M_{\mathrm{hf}}(2 S)_{b \bar{b}}=48.7 \pm 2.3 \pm 2.1 \mathrm{MeV}$. Our article, entitled "Observation of the $\eta_{b}(2 S)$ Meson in $\Upsilon(2 S) \rightarrow \gamma \eta_{b}(2 S), \eta_{b}(2 S) \rightarrow$ Hadrons and Confirmation of the $\eta_{b}(1 S)$ Meson" describing the above results has been recently published in the Phys. Rev. Lett. [6].

Since ours is the first observation of $\eta_{b}(2 S)$, it is interesting to compare it with the theoretical expectations. The latest unquenched lattice calculation by the HPQCD Collaboration [7] predicts $\Delta M_{\mathrm{hf}}(2 S)_{b \bar{b}}=35 \pm 3 \mathrm{MeV}$. This is $14 \mathrm{MeV}$ smaller than our result which is smaller still than the 

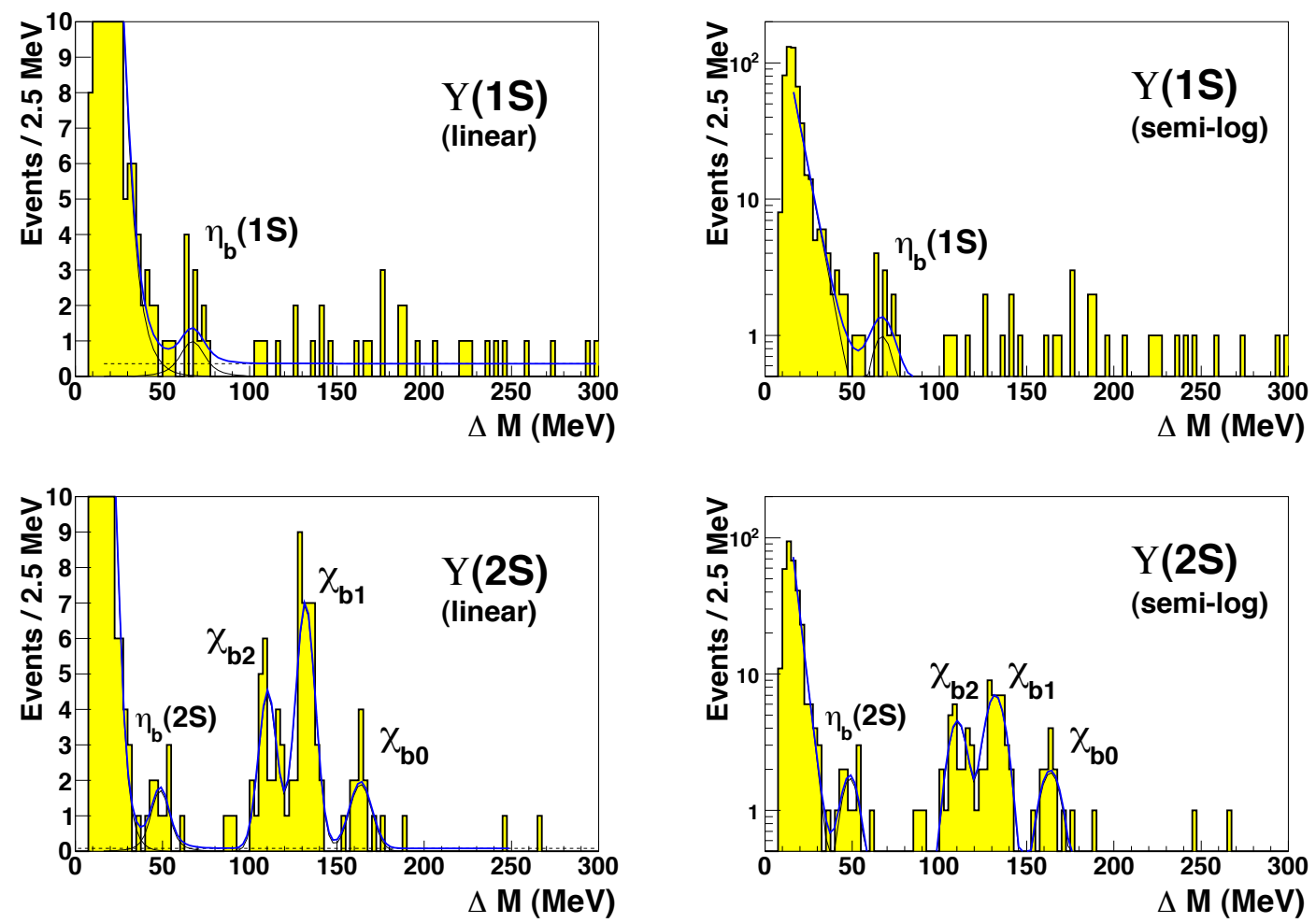

Figure 4: Fits to the $\Delta M$ mass distributions: (top row) $\Upsilon(1 S)$ linear and semi-log representations, (bottom row) $\Upsilon(2 S)$ linear and semi-log representations.

\begin{tabular}{l|c|c}
\hline & $\Upsilon(1 \boldsymbol{S}) \rightarrow \boldsymbol{\gamma} \boldsymbol{\eta}_{\boldsymbol{b}}(\mathbf{S})$ & $\Upsilon(2 \boldsymbol{S}) \rightarrow \boldsymbol{\gamma} \boldsymbol{\eta}_{\boldsymbol{b}}(\mathbf{2 S})$ \\
\hline$M\left(\boldsymbol{\eta}_{b}(n S)\right), \mathrm{MeV}$ & $9393.2 \pm 3.4 \pm 2.3$ & $9974.6 \pm 2.3 \pm 2.1$ \\
$\Delta M_{\mathrm{hf}}(n S)_{b \bar{b}}, \mathrm{MeV}$ & $\mathbf{6 7 . 1} \pm \mathbf{3 . 4} \pm \mathbf{2 . 3}$ & $\mathbf{4 8 . 7} \pm \mathbf{2 . 3} \pm \mathbf{2 . 1}$ \\
$\eta_{b}(n S)$ significance & $\sim 3 \sigma$ & $\sim 5 \sigma$ \\
$\mathscr{B}_{1} \times \sum_{i=1}^{26} \mathscr{B}_{2 i} \times 10^{6}$ & $\approx 30_{-7}^{+36}$ & $\approx 46_{-16}^{+31}$ \\
\hline
\end{tabular}

Table 2: Results from the analysis of $\Delta M(\Upsilon(1 S), \Upsilon(2 S))$ distributions of Fig. 4.

prediction of Meinel [8] of 23.5 $\pm 4.6 \mathrm{MeV}$. We have no explanation for these differences except to note that the two lattice calculations differ by more than $11 \mathrm{MeV}$, and lattice calculations are still evolving, and have admitted problems with radial excitations.

The peak which we observe at $\Delta M(\Upsilon(2 S))=48.7 \pm 3.1 \mathrm{MeV}$ has a log likelihood significance of $4.9 \sigma$, and we have assigned it as $\eta_{b}(2 S)$ because we can find no other possible explanation for it. If the existing lattice calculations are to be believed, its low mass is admittedly problematic. An explanation for it is needed. Is it an 'exotic', or is it $\eta_{b}(2 S)$ displaced by mixing with some exotic? Indeed, one such explanation exists in the literature. Domingo et al [9] have suggested that a light CP-odd Higgs Boson with the same $J^{P C}=0^{-+}$exists, and that it mixes with $\eta_{b}(2 S)$ to lower its mass from what is expected in absence of such mixing, resulting in larger $\Delta M_{\mathrm{hf}}$. This is illustrated 


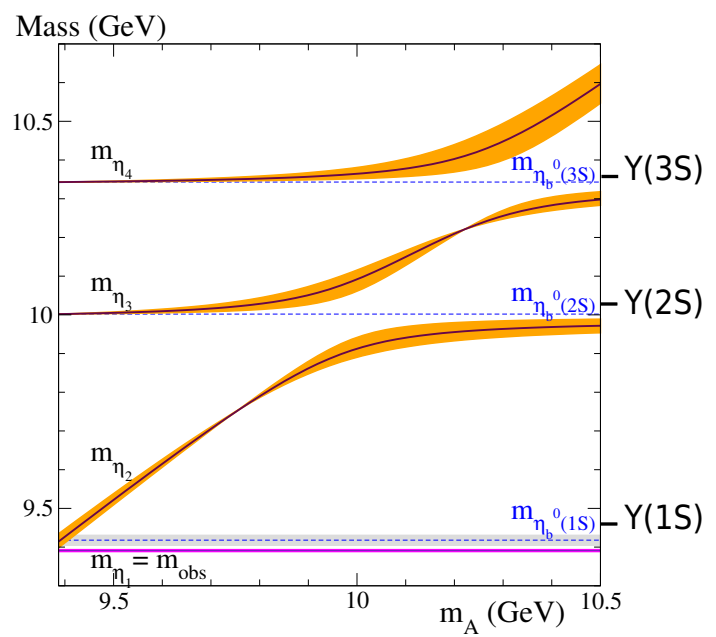

Figure 5: Illustrating the lowering of $M\left(\eta_{b}(n S)\right)$ due to mixing with a CP odd Higgs (from ref. [9]).

in Fig. 5. This possibility is admittedly speculative but highly exciting.

This investigation was done using CLEO data, and, as members of the former CLEO Collaboration, we thank it for this privilege. This research was supported by the U.S. Department of Energy.

\section{References}

[1] S. Godfrey and N. Isgur, Phys. Rev. D 32, 189 (1985).

[2] N. Brambilla, Y. Jia, and A. Vairo, Phys. Rev. D 73, 054005 (2006).

[3] S. Godfrey and J. L. Rosner, Phys. Rev. D 64, 074011 (2001).

[4] B. Aubert et al. (BaBar Collaboration), Phys. Rev. Lett. 101, 071801 (2008); B. Aubert et al. (BaBar Collaboration), Phys. Rev. Lett. 103, 161801 (2009).

[5] G. Bonavicini et al. (CLEO Collaboration), Phys. Rev. D 81, 031104(R) (2010).

[6] S. Dobbs et al. Phys. Rev. Lett. 109, 082001 (2012).

[7] R. J. Dowdall et al. (HPQCD Collaboration), Phys. Rev. D 85, 054509 (2012).

[8] S. Meinel, Phys. Rev. D 82, 114502 (2010).

[9] Domingo, et al. Phys. Rev. Lett. 103, 111802 (2009). 